Meta

Journal des traducteurs

Translators' Journal

\title{
Vers une vie nouvelle des langues
}

\section{Pierre Widal}

Volume 21, numéro 3, septembre 1976

URI : https://id.erudit.org/iderudit/004599ar

DOI : https://doi.org/10.7202/004599ar

Aller au sommaire du numéro

Éditeur(s)

Les Presses de l'Université de Montréal

ISSN

0026-0452 (imprimé)

1492-1421 (numérique)

Découvrir la revue

Citer cet article

Widal, P. (1976). Vers une vie nouvelle des langues. Meta, 21(3), 183-187.

https://doi.org/10.7202/004599ar d'utilisation que vous pouvez consulter en ligne.

https://apropos.erudit.org/fr/usagers/politique-dutilisation/ 


\section{Vers une vie nouvelle des langues}

Ausone, le Bordelais, ne parlait pas la langue du Sénéchal de Joinville qui ne s'exprimait pas comme Rabelais. Mais Rabelais ne se servait pas des mêmes termes que Montaigne dont le parler eût été archaïque au temps de Corneille. Là s'arrête la litanie. Brutalement, après mille années qui vont du latin décadent aux codifications de Malherbe, l'évolution est bloquée à qui ne sait pas le latin, ou l'aurait oublié, un glossaire est quasi indispensable pour lire un grand nombre des textes antérieurs au dix-septième siècle. Dès le Cid, par contre, tout est immédiatement compréhensible, voire usuel.

Le phénomène est général et quasi simultané. L'anglais, l'allemand, après Shakespeare, avant Schiller, eux aussi se sont figés. Il en est de même en Espagne et en Italie. Les langues occidentales ont évolué pendant un millénaire, plus ou moins rapidement, plus ou moins régulièrement, mais indéniablement, puis, subitement, il y a eu fixation. On peut dire à propos du français, que la dernière invasion culturelle, celle des mots italiens, s'est arrêtée aux portes de l'Hôtel de Rambouillet. $\mathrm{Et}$, pendant quelque trois cents ans, mis à part quelques rares néologismes, les langues vivantes se sont comportées comme des langues mortes.

Une explosion nouvelle les secoue aujourd'hui et l'on voit partout aux vitrines des libraires de toutes les capitales, s'aligner les titres d'ouvrages qui dénoncent le massacre de nos héritages culturels respectifs. Le français s'anglicise, mais envahit l'allemand, s'infiltre aux États-Unis et en Italie, bref, on sonne l'alarme chez tous ceux qui n'admettent pas et dénoncent la surprenante et omniprésente reprise d'une évolution linguistique, unanimement jugée abâtardissante.

Que s'est-il donc passé il y a trois cents ans? Que se passe-t-il donc aujourd'hui ? Le phénomène fut général, il l'est de nouveau. Il a fallu que se produise un fait particulier ou un ensemble de circonstances dont l'influence semble s'être effacée. On peut essayer peut-être d'expliquer par des considérations historiques cette stabilité insolite, son début et sa fin.

In principio erat verbum. Les gens parlaient, les savants lisaient et écrivaient. Ils étaient l'élite, mais leurs carrés ne pouvaient résister aux bataillons innombrables des illettrés. Les tendances évolutives avérées, selon des mécanismes bien connus, interventions, simplifications, fautes de prononciation, toutes les techniques diverses longuement décrites de l'évolution des langues parlées, s'imposaient petit à petit. 
Il fallait se résigner à les admettre pour être compris, accepter et utiliser ces innovations qui ont fait passer du latin, langue universelle aux divers stades du français. Ces transformations ont existé de tout temps. Les hiéroglyphes ont cédé la place à des écritures plus simples, le latin de Tite Live s'est avachi dans le bas-latin ecclésiastique, exemple rituellement cité dans les classes d'humanités. Rien d'étonnant donc à ce que de «niger» on en arrive à «nigrum» pour finir avec «noir». Il serait inutile de s'attarder davantage à ce processus bien connu, s'il s'était poursuivi au cours des trois cents dernières années. Ce qui est remarquable, c'est qu'il ait subsisté, presque comme un cheminement souterrain d'eaux d'infiltration, en abrégeant des mots nouveaux courants comme taximètre devenu «taxi $»$. Mais ces modifications sont minimes et tiennent bien souvent plus à l'érosion prévisible des valeurs verbales qu'à une mutation constante et vigoureuse. Celle-ci a indiscutablement cessé.

Dans le siècle qui a précédé cet arrêt, une invention a permis une incroyable diffusion des mots utilisés et la mise à la disposition de masses sans cesse croissantes, de formes stabilisées, puisque imprimées. Assurément, Gutenberg en fondant ses premiers caractères a immobilisé une certaine façon de s'exprimer, dans toutes les civilisations occidentales. On peut dire, sans exagérer, que l'imprimerie a créé une paralysie.

Il a naturellement fallu du temps pour que ce frein fasse effet. Il est normal que la connaissance du langage imprimé ne se soit pas répandue comme une épidémie de peste noire. On devra attendre jusqu'en 1650 pour que la majorité des classes agissantes soit gagnée au savoir élémentaire de la lecture et de l'écriture. En cent cinquante ans, les moines, les médecins, les avocats, les marchands, les financiers et les premicrs entrepreneurs se sont vus dans l'obligation de rédiger et de comprendre les messages d'un savoir transmis à des centaines, voire à des milliers d'exemplaires. Quand on puise la connaissance dans les livres, on est à l'abri des erreurs des copistes, on corrige d'après les précédents les déformations du langage parlé et l'on s'en tient à des normes verbales qui permettront d'être compris par ses pairs. C'est la première fois que l'écrit dicte sa loi à la parole.

L'on peut, dans les discussions, démontrer son érudition en citant des références accessibles immédiatement, s'appuyer sur l'autorité des écrits «C'est vrai puisque c'est imprimé », et, désormais, s'instaure le respect de cet imprimé. Les lettrés vont même pouvoir se moquer des ignorants et écrire pour l'amusement des raffinés les romans picaresques où l'on fait parler les paysans ou les bateliers, en plaisantant leur accent et, mais oui ! leurs fautes. Et tout un public en rira.

Il reste un pas à franchir. Pour immobiliser définitivement les langues dans le corset de fer de leur perfection, il faut fixer des règles de grammaire et sanctifier l'orthographe. La chose se fera simultanément et l'on ne verra plus un maréchal de Richelieu écrire «la cuisin » ou «les page» en 1760 sous le sourire bienveillant d'une aristocratie qui $n^{\prime} y$ trouvait que la force de caractère d'un grand seigneur, impatient du joug de ses précepteurs dès sa plus petite enfance. L'empire va mettre bon ordre à ces fantaisies et l'apogée de l'orthodoxie n'est pas loin. Au nom du droit imprescriptible au savoir, l'école sera obligatoire donc généralement gratuite, et objective, donc laïque, dans certains pays tout au moins. 
Enfermés autour du préau, les petits enfants apprendront, de 5 à 12 ans, les éléments indispensables à la conduite judicieuse et morale d'un individu éclairé dans un monde de plus en plus complexe. Ils sauront lire les lois et n'auront aucune excuse valable pour les ignorer. Elles s'appliqueront dès leur publication et il n'y aura plus de tambours de ville. Dès l'invention des aéroplanes, ils sauront qu'il ne faut pas dire un « aréo» comme s'obstinent à le faire leurs grands-parents attardés. Lorsque la crise cardiaque aura reçu ses lettres de noblesse et été promue à la dignité d'infarctus, l'«infractus » n'aura duré que le temps d'un éclair et le moindre bouvier des campagnes les plus reculées s'empressera de contracter ses maxillaires pour «prononcer correctement, comme ça s'écrit ».

Que se passe-t-il donc aujourd'hui et pourquoi les candidatures deviennentelles des applications, la détente, du relaxe, un butler, le «maître d' » et comment fleurit-il autant de merchandising en français que de «savoir faire » ou d' « esprit de corps $\gg$ en anglais ou en américain ? Pourquoi un parfumeur français fait-il paraître une annonce en anglais dans un hebdomadaire parisien tandis qu'un importateur paye une page entière en français dans une des revues les plus lues des Etats-Unis?

Faut-il invoquer l'opulence des sociétés de consommation et les enrichissements culturels que nous valent les formidables transplantations de population nées du tourisme estival?

Assurément, le phénomène joue son rôle dans le brassage verbal. L'internationalisation se diffuse dans tous les domaines. L'Europe mange des hamburgers et des hot dogs, pendant que de l'autre côté de l'Atlantique, on se régale de «pizzas » et de «casserole», voire d'hibachis. Évidemment, ces énormes migrations annuelles qui déferlent pour refluer trois mois plus tard ne sont pas comparables aux misérables cohortes romaines ou aux quelques centaines de cavaliers wisigoths - tout juste de quoi remplir trois ou quatre 747 - dont la simple présence suffisait à latiniser l'Occident ou à enrichir de termes germaniques le parler toulousain. L'effet $\mathrm{du}$ tourisme lui n'est pas durable, à première vue, tout au moins. Il faut se rappeler cependant que de juin à septembre, c'est tous les ans qu'il se reproduit. Outre ses effets économiques, passagers mais chiffrables, il laisse sur la berge des détritus et en emporte d'autres, véritable épidémie d'un verbalisme international. Où, dans l'univers tout entier, des Fidji aux Îles Lofoten, le mot «film» ne sera-t-il pas compris ? Et « hôtel », et «bar » et «star » ? Et pour qui le nom de Ford n'évoquet-il rien? Entre les féodaux, le clergé a imposé au bout de quelques siècles la trêve de Dieu. Faudra-t-il aussi longtemps pour que se crée un sabir international qui a apporté «drugstore » à Paris et, paraît-il, la « détente » dans le monde ?

Ce ne serait tout de même pas suffisant. Les prodigieuses découvertes d'un siècle qui a permis de passer de la bougie au congélateur et de la diligence à la fusée interplanétaire ont eu leur effet sur notre façon de nous exprimer. On ne peut pas parler des cosmonautes dans le langage de Cyrano de Bergerac, ni non plus, quand même, dans celui de Jules Verne. Les différents jargons scientifiques ont connu une diffusion sans précédent. On peut affirmer que dans l'hémisphère boréal tout entier, on sait quel ampérage un appareil ménager doit prélever et sous 
quel voltage. Il est aussi inimaginable qu'un automobiliste, quelque fruste qu'il soit, ne connaisse pas la pression de ses pneus ou la nécessité d'une vidange, qui ne se fera pas grâce aux bons soins d'un vidangeur.

Il y a là des acquisitions terminologiques massives et aisément explicables, mais du fait même de leur absorption, elles ont été nationalisées. Les racines sont restées les mêmes. Il y a «moteur», «motor», "motore». On trouve «car», 《voiture » ou «machine» à côté des formes «automobile». Pour toutes ces notions, il y a assimilation et tout porte à croire que cette tendance conservera sa vigueur dans les mots d'emploi quotidien.

Le problème, en réalité, est ailleurs. Il se centre non pas même sur «merchandising », « marketing » ou « input », mais dans l'emploi vicié de mots étrangers à la place des termes de sens identique existant dans la langue contaminée. "Une application » est une candidature, des «fonds mutuels» sont des fonds mis en commun, une "partition », même bruyante, cesse d'être musicale pour devenir une sécession ou une scission, consécutive à des complications militaires ou diplomatiques. Les exemples de ces intrusions sont innombrables, dans toutes les langues. On en relève dans les journaux à chaque instant et il n'en manque pas dans les revues mêmes, qui se piquent de purisme et de correction linguistique. Quels que soient les responsables, traducteurs incompétents ou rédacteurs qui confondent autorité et prétention, peu importe, le fait est là et les mots défectueux sont de plus en plus utilisés et acceptés, le savoir a-t-il changé de contenu, ou les correcteurs ne savent-ils plus accomplir leur travail ?

Il semble au vrai qu'il faille incriminer la diffusion même des connaissances de base. Il y a tant de choses à apprendre, tant d'événements qui surgissent, que l'esprit, comme une mouche, se cogne à la vitre de l'assimilation. Un homme sur la lune, une protestation de la moitié féminine du genre humain contre l'oppression qui l'accable depuis la première pomme, les fumées délétères des usines, une révolution par-ci, une fin du monde par-là, les loisirs d'un homme occupé ne suffisent pas à les lui faire appréhender intellectuellement sous forme d'une lecture génératrice de réflexion. Mais, il y a un moyen facile de savoir l'essentiel. Il est électronique, de manipulation élémentaire et suffisamment accessible pour se répandre partout. Qu'il s'agisse d'ondes sonores ou lumineuses, le niveau de leur message prédigéré et souvent orienté est assez bas pour être aisément perçu par la «clientèle de masse », anciennement la masse de la clientèle. Leur importance est telle qu'aucun politicien lucide n'acceptera de faire un discours ou de diriger une manifestation à un moment où l'on diffuse un événement sportif d'importance. Et, là, maintenant, on ne lit pas, on écoute ou on regarde.

Le fait est capital. La parole a repris sa priorité, l'imprimé recule. À quoi bon se donner le mal de lire, de choisir, de penser, quand il suffit d'écouter. Tout est présenté clairement, vulgarisé, jugé en même temps qu'expliqué. L'objectivité, l'honorabilité sont des données présumées acquises au départ. Il n'y a pas d'émission d'information qui paraisse doctrinale, comme l'est la tendance de l'une ou l'autre presse. Le prestige d'une nouvelle importante rejaillit sur le «speaker ». Ainsi, un comprimé remplace une cure longue et difficile. Ce n'est pas ici le lieu d'épiloguer 
sur les répercussions non linguistiques du phénomène. Mais l'incidence sur le langage est immense. D'abord les vulgarités qui avaient disparu des écrits, surgissent à nouveau, signes avant-coureurs de l'évolution de l'expression. En outre, une dépêche traduite à la dernière seconde contient une formule fautive. Quelques millièmes de secondes plus tard, une population entière a entendu, compris, emmagasiné. Elle la répétera, on peut en être certain. Par ailleurs, les formes un peu compliquées, même énoncées correctement vont être entendues et non vues. À la limite, on en arrive à l'orthographe phonétique ou peut-être à une langue nouvelle, encore inconnue, où l'Amphytrion ne serait pas celui de Plaute, ni les Fables celles que disait Esope...

Il faut savoir s'incliner devant la puissance du temps. Il y eut Euripide, mais il y a eu Racine, voire Henry Bataille. Il y a eu des chaises curules, puis des bergères Louis $\mathrm{XV}$ et enfin des fauteuils «profonds comme des tombeaux ». Le coureur de marathon est remplacé par des satellites, plus rapides, il faut le concéder. Faut-il désespérer de cette mutation des langues? Elles étaient tout de même bien différentes, il y a quelques années, du parler des glorieux ancêtres. De tout temps, les puristes, laudateurs temporis acti ont été bruyants et désespérés. L'effet de leurs clameurs, avouons-le, a été à peu près nul. Il semble bien que nous soyons à la veille d'assister à la disparition d'une forme de perfection précieuse et exquise. Nous le constatons le cour serré. Mais est-ce une raison pour maudire - tout à fait en vain du reste- les torrentielles audaces verbales d'un Céline ou d'un Henry Miller ? Un parler nouveau est en gestation, né d'un embryon commun. Il annonce peut-être des communications et une compréhension plus universelles, l'élimination aussi de valeurs culturelles sans prix. Nul ne peut s'opposer au flot des barbares, ni au flux des barbarismes. Comme le disait déjà Horace "Multa ceciderunt quae nunc sunt in honore verba... » et les civilisations savent désormais qu'elles sont mortelles.

Pierre Widal 